Original article

\title{
Epidemiology and clinical features of human cystic echinococcosis in adults from an endemic area (Morocco)
}

\author{
Omar Amahmid ${ }^{\mathrm{a}, \mathrm{b}, \mathrm{c}, *}$, Youssef El Guamri ${ }^{\mathrm{a}, \mathrm{c}}$, Khalid Zenjari ${ }^{\mathrm{d}}$, Souad Bouhout ${ }^{\mathrm{e}}$, Mohamed Ait Moh ${ }^{\mathrm{f}}$, \\ Fatima Boraam $^{\mathrm{f}}$, Abdelaziz Ait Melloul ${ }^{\mathrm{g}}$, Hilal Benfaida ${ }^{\mathrm{g}}$, Khadija Bouhoum ${ }^{\mathrm{b}}$, Driss Belghyti ${ }^{\mathrm{c}}$ \\ ${ }^{a}$ Biology Unit, Department of Life and Earth Sciences, Regional Centre for Careers of Education and Training (CRMEF-Marrakech), Marrakesh, Morocco \\ ${ }^{\mathrm{b}}$ Parasitology Unit, Department of Biology, Laboratory of Hydrobiology, Ecotoxicology and Sanitation (LHEA), Faculty of Sciences-Semlalia, Marrakesh, Morocco \\ ${ }^{c}$ Department of Biology, Laboratory of Biotechnology, Environment and Quality (LABEQ), Faculty of Sciences, Kenitra, Morocco \\ ${ }^{\mathrm{d}}$ Regional Direction of Health Marrakesh-Safi, Marrakesh, Morocco \\ ${ }^{\text {e }}$ Service of Parasitic Diseases, Direction of Epidemiology and Diseases Control (DELM), Rabat, Morocco \\ ${ }^{\mathrm{f}}$ Service of Public Health and Epidemiological Surveillance, Regional Direction of Health, Marrakesh, Morocco \\ ${ }^{\mathrm{g}}$ Regional Laboratory of Epidemiological Diagnostic and Environmental Hygiene (LRDEHM), Marrakesh, Morocco
}

\section{A R T I C L E I N F O}

\section{Keywords:}

Adults

Clinical features

Cystic echinococcosis

Cyst location

Risk factors

\begin{abstract}
A B S T R A C T
Background: Cystic echinococcosis is a parasitic disease of major public health concern in many countries. Infection is generally acquired during childhood and symptoms may appear later in adulthood. The study aimed to determine the pattern and characteristics of cystic echinococcosis in adults from an endemic area (Morocco), where a control and prevention program has been implemented.

Methods: Adult patients diagnosed and operated for echinococcosis between 2010 and 2016 were investigated. Reports containing details regarding each patient were established and completed.

Results: Over the study period, 260 patients were investigated with a decreasing trend in patients' numbers. Adults from rural areas were significantly predominant $(74.2 \%)(\mathrm{p}<0.01)$. Females were more infected $(55.8 \%)$ than males $(44.2 \%)$. Adults aged 18-43 years were the most affected $(52.3 \%)(\mathrm{p}<0.01)$. All of the patients had single organ involvement. Liver location was preponderant $(63.8 \%)$ and patients with uncommon locations were rare (6.9\%). Cysts' distribution varied by gender, age group and origin. Mean length of hospital stay was 5.5 days, with relatively higher periods in patients aged 57-82 years and in patients with unusual locations of cysts.

Conclusion: Active transmission of cystic echinococcosis can still occur in areas where surveillance programs have been implemented. Many factors affect features of the disease and should be considered in prevention and control programs in endemic areas.
\end{abstract}

\section{Introduction}

Human cystic echinococcosis (CE) or hydatidosisis is a common zoonotic disease affecting more than one million people worldwide. ${ }^{1} \mathrm{CE}$ does not remain restricted to endemic geographical areas anymore but rather is a global health concern, causing significant morbidity and mortality with severe economic losses. ${ }^{2}$ Human infection occurs by ingesting eggs of Echinococcus granulosus, shed in the faeces of infected canines. The main common routes of humans infection are direct contact with definitive host, ingestion of contaminated water and food sources, or direct feco-oral transmission.

Although control programmes for $\mathrm{CE}$ have been implemented in many endemic regions worldwide to reduce or eliminate $\mathrm{CE}$, the disease continues to be a significant public health problem. Furthermore, in several countries $\mathrm{CE}$ is considered as an emerging or re-emerging zoonose with an increasing trend in hydatid cyst cases. ${ }^{3}$ In addition, CE has been included within the group of neglected tropical diseases. ${ }^{4}$ Neglect impedes the collection of good-quality data for prevention measures and control programmes implementation. To fill this gap epidemiological surveys are recommended as part of an effective control approach and studies on regional characteristics of $\mathrm{CE}$ offer valuable data that could be the base for the implementation of public health policies in endemic areas such as Morocco and surrounding countries. ${ }^{5}$

$\mathrm{CE}$, which commonly starts during childhood and described as a

\footnotetext{
* Corresponding author. Biology Unit, Department of Life and Earth Sciences, Regional Centre for Careers of Education and Training (CRMEF Marrakesh), P.O. Box 797, Mozdalifa Street, Marrakesh, 40090, Morocco.

E-mail addresses: amahmid1969@gmail.com, amahmid@hotmail.com (O. Amahmid).
} 
young adult disease, may be observed at any age. The incubation period of the disease is often prolonged for several years and infection mostly occurs at an early age remaining asymptomatic until the cysts reach a large enough size to cause dysfunction. ${ }^{6}$

Several studies have been focused on cystic echinococcosis in children and it has been suggested that in areas where the prevalence of hydatid cyst in adults is high, this prevalence is also high in children. ${ }^{7-9}$ However, published researches regarding the pattern and characteristics of human CE involvement in adults are rare. The current study aimed to explore the trend and features as well as risk and exposure factors of CE in adults living in an endemic area in Morocco.

\section{Methods}

The undertaken study included 260 adults aged $\geq 18$ years diagnosed and treated for CE during a seven-year period (2010-2016) at two major referral governmental hospitals (Ibn Zohr and Ibn Tofail) in Marrakesh (Morocco). These hospitals are ranked among the main consultation centres for patients from several primary healthcare centres and provide medical care for patients hailing from many provinces of the area. For the diagnosis of CE among the investigated adults, imaging techniques, including ultrasonography, computed tomography and standard radiology, have been used. All of the patients have been operated surgically and the most privileged surgical approaches consisted of laparotomy, thoracotomy and craniotomy. Medical treatment was scheduled in conjunction with surgery. ${ }^{10}$ Albendazole was used prior to the surgical operation and after surgery, or administered only after surgery. The excised cysts were sent for histopathological examination for differentiation and confirmation of diagnosis.

The data collection was conducted using a report containing all the details regarding each $\mathrm{CE}$ case. Collected information included, age, gender, place of residence (rural versus urban), date of admission, date of leave, diagnosis techniques, location of the cyst(s) and treatment method. The tendency of CE cases during the study period has been described. Data were analyzed according to adults' place of residence (urban or rural), age and gender. The distribution of cysts in adults has been investigated according to anatomical site and patients' origin. The length of hospital stay was determined and assessed by patient's age and anatomic location of cysts.

Descriptive statistical analysis was performed to assess variables and trends (e.g. mean numbers, medians, standard deviation and proportions). Trends over time for the infection rates of adults were determined by linear regression analysis. The Chi-square test was used to determine the associations between the various potential risk factors like age, sex, year variation and comparison of the occurrence frequencies and distribution. Student's t-test was used to compare the men length of hospital stay according to age groups and anatomic location of cysts. All data were analyzed using Excel and SPSS software (version 17 ), with a p-value $<0.05$ was considered as statistically significant.

\section{Results}

Over the seven-year study period 260 adults ( $\geq 1$ 8years) residing in several provinces have been diagnosed and treated for cystic echinococcosis (Fig. 1). The number of CE cases ranged from 22 cases in 2012 to 54 cases in 2010, with an average of $37.1 \pm 12.5$ cases per year; $27.6 \pm 10.3$ in rural area versus $9.6 \pm 2.6$ in urban area. An overall, but non-uniform, decreasing tendency in $\mathrm{CE}$ frequency was recorded over the study period $\left(\mathrm{R}^{2}=0.28, \mathrm{p}>0.05\right)$ (Fig. 2).

The distribution of $\mathrm{CE}$ cases by place of residence (rural versus urban), gender and age is presented in Table 1 . The study results showed that $74.2 \%$ (193 cases) of the 260 adults were rural residents while $25.8 \%$ (67 cases) originated from urban areas, and the observed difference was statistically significant $(\mathrm{p}<0.001)$. For gender, CE frequency was higher, but not significantly, in females than in males, with respective proportions of $55.8 \%$ (145 cases) and $44.2 \%$ (115 cases) and male to female infection ratio of 0.79:1 ( $p>0.05)$. Both males and females hailed from rural environs were significantly more affected than those living in urban areas $(p<0.001)$. With respect to age factor, the investigated adults were aged between 18 and 82 years. The mean age of patients was $38.9 \pm 15$ years, with a median age of 36 years. The distribution of $\mathrm{CE}$ cases by age-groups registered a general decreasing trend of $\mathrm{CE}$ frequency with age (Table 1). Indeed $52.3 \%$ of patients were aged $18-25$ years versus $11.5 \%$ for $57-82$ years aged group. Therefore, young adults were more likely to get infected than age groups over 44 years old $(p<0.01$ ). Additionally, for all the age groups rural residents were highly affected by $\mathrm{CE}$ than urban residents $(p<0.05)$.

The investigation of the anatomic location of cysts showed that single organ involvement was observed in all the 260 adults while none of the patients had multiple organs affected (Table 2). Liver localization of cysts was significantly more preponderant than lung cysts with respective rates of $63.8 \%$ (166 cases) and $29.2 \%$ (76 cases). The liver to lung infection ratio was 2.18:1 ( $\mathrm{p}<0.001$ ). Involvement of sites other than liver and lungs was rare (18 cases, 6.9\%); four adults (1.5\%) had brain cysts, each of dorsal subcutaneous and kidney locations were identified in three cases $(1.1 \%)$, two cases $(0.8 \%)$ had cysts in vertebrae and a single case for urinary bladder, mediastinum, medullar, pelvic cavity and peritoneum locations $(0.4 \%)$. No recurrences have been recorded.

In relation to the distribution of cysts by patients' origin, all of the cyst locations were more frequently detected in adults hailed from rural areas as compared to urban residents $(p<0.001)$. For the gender factor, liver cysts were significantly more identified in females than in males $(\mathrm{p}<0.01)$ while no statistically significant differences were observed for lung and unusual locations ( $p>0.05$ ). For all of the defined age groups liver location was significantly frequent than lung and uncommon locations ( $\mathrm{p}<0.01$ ). By age groups, liver and lung locations occurrence was not significantly different between 18-30 and 31-43 years aged patients while a significant decrease was observed in older age groups $(\mathrm{p}<0.01)$. The unusual cyst location was significantly preponderant in adults of 31-43 years (Table 2).

The median length of hospital stay was 4 days with an average of 5.5 days and a range of 1-65 days (Table 3). The analysis for trend showed an overall increase in the hospitalization duration with age. Relatively higher periods were recorded in 57-82 years aged adults (7 days) compared to those aged 18-30 years (4.8 days). Additionally, the mean length of hospital stay was relatively higher in patients with uncommon cyst locations (7.8 days) as compared to liver and lungs locations (5.5 and 4.5 days, respectively) (Fig. 3). However, the Student's t-test did not show any significant difference between the lengths of hospital stay by patients age and cyst locations ( $p>0.05$ ).

\section{Discussion}

Cystic echinococcosis is a zoonotic disease with a wide geographical distribution, engendering economic losses and major public health concern in many endemic countries. ${ }^{1}$ Despite this global burden, CE remains a neglected zoonosis. ${ }^{4}$ The disease prevails in all age groups and new infections occur in children and cysts may persist and grow for many years before manifestations are seen. ${ }^{6}$ In Morocco, as CE continues to be a significant public health problem an active surveillance has been undertaken in the country for the disease control. ${ }^{11}$ In this context research studies may provide useful epidemiological information on the prevailing situation for CE in the studied areas. During the seven-year study period, a mean number of 37.1 of CE cases per year was recorded. For similar study periods, higher average numbers of CE cases in adults have been reported in other endemic areas undergoing control programs, reaching 52.7 cases per year in Western Romania and 52.3 cases per year in Uruguay, respectively. ${ }^{12,13}$ The number of reported CE cases in this study may not reflect the real occurrence of the disease due to underreporting. A proportion of patients may attend 


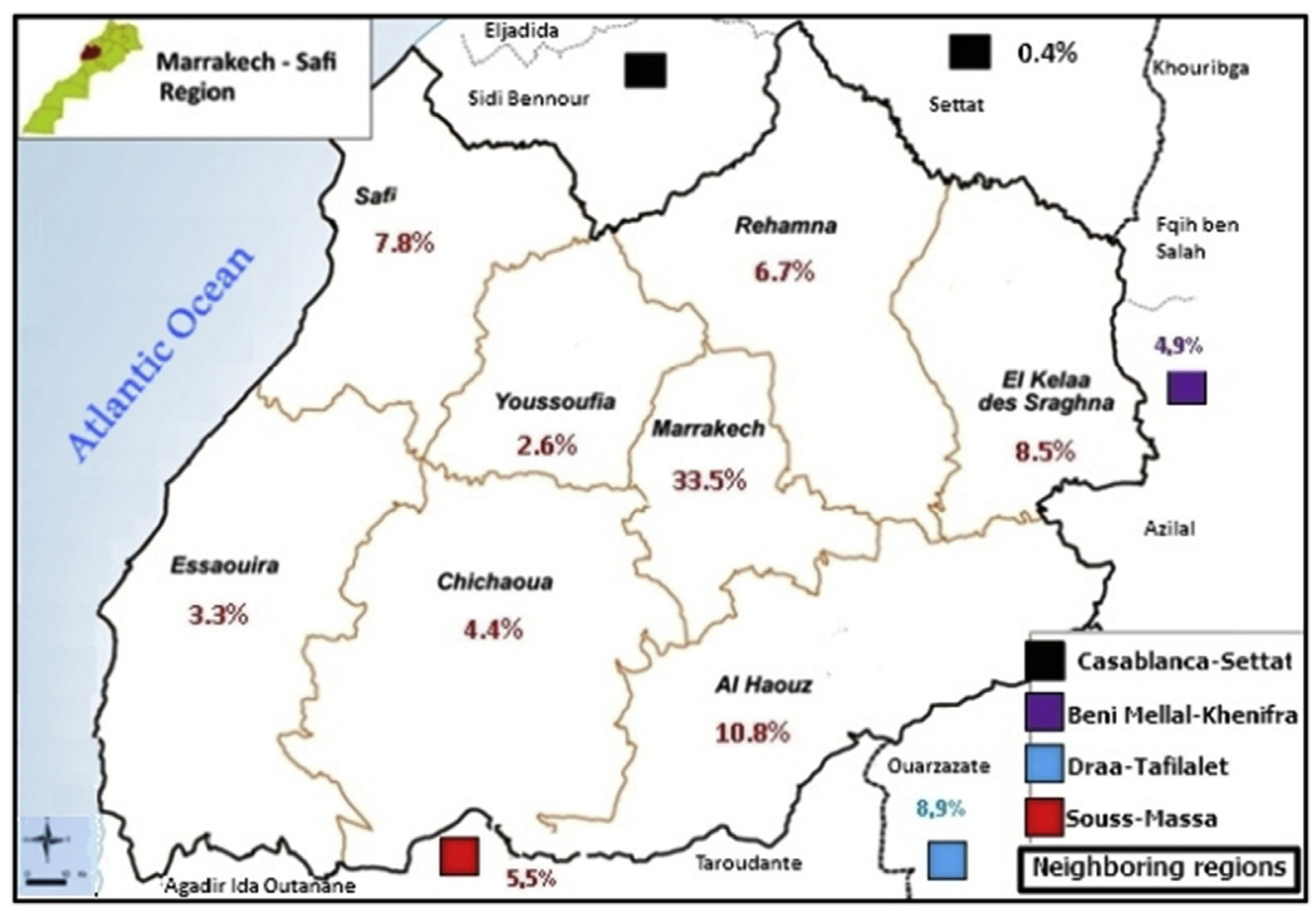

Fig. 1. Geographical origin of the adults diagnosed and operated for cystic echinococcosis during the study period (2010-2016).

private hospitals or refer to other hospitals out of the area. ${ }^{14}$ Moreover, $\mathrm{CE}$ is often asymptomatic and affects communities with limited access to health facilities. ${ }^{2,5}$ The present study showed a descending tendency in the CE occurrence in adults through the seven-year study period. This finding is in accordance with the results of other authors indicating a non-uniform decline in $\mathrm{CE}$ infection rates over time in adult patients as a result of national control programs. ${ }^{12,13}$

For the patients' origin, adults hailed from rural environs were significantly more affected by CE (74.2\%) than urban residents (25.8\%). In rural residency the main risks factors for $\mathrm{CE}$ in the investigated cases are dog ownership and the close proximity and cohabitation of owned-dogs with their owners and livestock. Studies carried out on owned dogs in rural localities found that $38 \%$ of dogs were infected. ${ }^{15,16}$ These dogs can easily spread large numbers of Echinococcus eggs on the agricultural fields, vegetables and water where they can survive and remain infective for months. In rural environs, the main occupations of adults are herding livestock and farming. Water,
Table 1

Numbers and proportions of cystic echinococcosis cases by place of residence, gender and age in adults diagnosed and treated for hydatid cysts.

\begin{tabular}{clll}
\hline \multicolumn{5}{c}{ Rural environment $\mathrm{n}(\%)$} & Urban environment n(\%) & Total n(\%) \\
\hline $\begin{array}{l}\text { Cases } \\
\text { Gender }\end{array}$ & $193(74.2)$ & $67(25.8)$ & $260(100.0)$ \\
Males & $89(34.2)$ & & \\
Females & $104(40.0)$ & $26(10.0)$ & $115(44.2)$ \\
Age groups (years) & $41(15.8)$ & $145(55.8)$ \\
$18-30$ & $69(26.5)$ & & \\
$31-43$ & $67(25.8)$ & $20(7.7)$ & $89(34.2)$ \\
$44-56$ & $27(10.4)$ & $21(8.1)$ & $43(33.9)$ \\
$57-69$ & $21(8.1)$ & $16(6.2)$ & $31(11.9)$ \\
$70-82$ & $9(3.5)$ & $10(3.8)$ & $9(3.5)$ \\
\hline
\end{tabular}

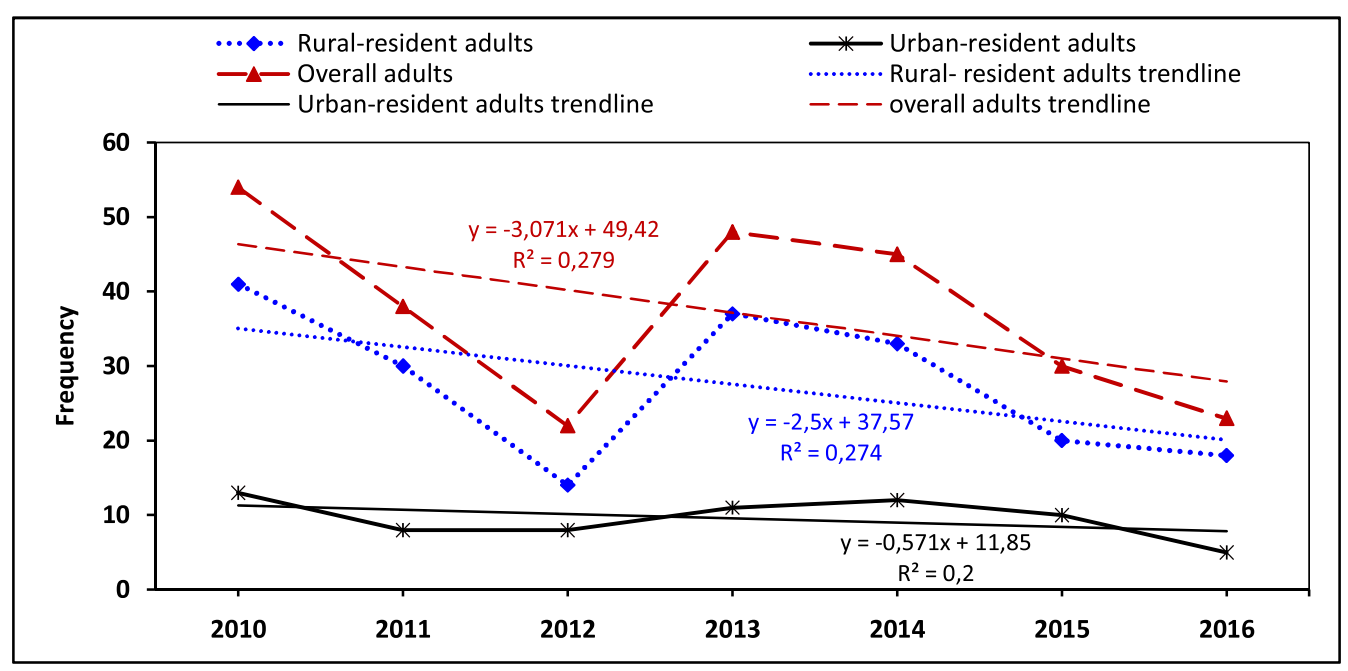

Fig. 2. Distribution and trends overtime of cystic echinococcosis cases in adults diagnosed and operated for hydatid cysts during the study period (2010-2016). 
Table 2

The anatomic location of cysts by gender, age and environment type in cystic echinococcosis adult patients.

\begin{tabular}{lllll}
\hline \multicolumn{5}{l}{ Cyst locations n(\%) } \\
\cline { 2 - 5 } & Liver & Lung & Uncommon & Total \\
\hline $\begin{array}{l}\text { Total Cases } \\
\text { Gender }\end{array}$ & $166(63.8)$ & $76(29.2)$ & $18(6.9)$ & $260(100.0)$ \\
Males & $66(25.4)$ & $41(15.8)$ & $8(3.1)$ & $115(44.3)$ \\
Females & $100(38.5)$ & $35(13.5)$ & $10(3.8)$ & $145(55.8)$ \\
Age groups (years) & $57(21.9)$ & $29(11.2)$ & $3(1.2)$ & $89(34.3)$ \\
$18-30$ & $48(18.5)$ & $29(11.2)$ & $11(4.2)$ & $88(33.9)$ \\
$31-43$ & $31(11.9)$ & $10(3.8)$ & $2(0.8)$ & $43(16.5)$ \\
$44-56$ & $23(8.8)$ & $8(3.1)$ & $0(0.0)$ & $31(11.9)$ \\
$57-69$ & $7(2.7)$ & $0(0.0)$ & $2(0.8)$ & $9(3.5)$ \\
$70-82$ & & & & $193(74.2)$ \\
Environment & $119(45.8)$ & $58(22.3)$ & $16(6.1)$ & $67(25.8)$ \\
Rural & $47(18.1)$ & $18(6.9)$ & $2(0.8)$ & \\
Urban & & & & \\
\hline
\end{tabular}

Table 3

The lengths of hospital stay according to age and cyst location in adult patients surgically treated for cystic echinococcosis.

\begin{tabular}{lll}
\hline & Average (days) & Median (days) \\
\hline Age groups (years) & & \\
$18-30$ & $4.8 \pm 4$ & 4 \\
$31-43$ & $5.4 \pm 6.7$ & 4 \\
$44-56$ & $5.7 \pm 4.3$ & 4.3 \\
$57-82$ & $7.0 \pm 10.1$ & 5 \\
Cyst location & & \\
Liver & $5.5 \pm 5.7$ & 4 \\
Lungs & $4.5 \pm 3.2$ & 4 \\
$\quad$ Uncommon & $7.8 \pm 14.3$ & 5 \\
Overall & $5.5 \pm 6.2$ & 4 \\
\hline
\end{tabular}

soil and crops contaminated with infected dog faeces increase the risk of human and animal infection and perpetuates CE transmission. ${ }^{1,17,18}$ In ruminants, an infection rate ranging from $1.9 \%$ in goats to $23.0 \%$ in cattle has been found ${ }^{19}$; whereas in humans an average of 5.2 surgical cases per 100,000 inhabitants have been reported, with uneven regional distribution. ${ }^{11}$ Yet the actual occurrence of CE in humans may be underestimated since a high proportion of cases remain asymptomatic for many years and, even when symptomatic, may never reach medical attention or official disease records. ${ }^{20}$

Other risk factors that may increase prevalence in rural residents include low education levels, poor economic conditions and medical services. In a previous survey, the assessment of the main attitudes and practices that promote $\mathrm{CE}$ transmission, have shown that about $50 \%$ of the investigated persons have not heard of $\mathrm{CE}$, and $78.7 \%$ of them were not aware of the dog's role in the disease transmission. Practices such as allowing dogs to roam around the family home, give dogs access to the kitchen as well to livestock housing were common. ${ }^{15}$ The limited education level of the rural population, and poor knowledge of transmission risks is reflected in engagement in risky behaviours such as mismanagement of infected ruminant organs and poor hygienic practices. ${ }^{13}$ Dogs use to roam around the slaughterhouses where they have easy access to discarded infected organs, considering the neglectful practices and unawareness of slaughterhouse workers. Similarly, uncontrolled home slaughtering during religious or local festivities is very common and the infected offal unsuitable for consumption are discarded and consumed by dogs. The findings of the current study are consistent with previous reports indicating that $\mathrm{CE}$ is more common in rural areas. ${ }^{14,21}$

In urban residency, CE has been mainly diagnosed in patients originated from surrounding peri-urban areas or having a repeated travel history to rural high-risk areas where suitable conditions for CE transmission are encountered. Furthermore, migration into urban localities creates peripheral settlements where rural-like risk factors prevail. These are considered as sources of infection to neighbouring people in the urban environment. Increasing numbers of free-roaming owned and stray dogs can move freely between peri-urban settlements and urban districts. Infected dogs shedding E. granulosus eggs in urban public spaces (gardens, parks) and contact with dogs, represent a potential risk factor for human CE transmission. ${ }^{22,23}$ Previous reports revealed an extension of CE in the urban zones. The occurrence of CE was found to be significantly higher in urban residents as compared to rural residents in studies carried out in Serbia and Tunisia. ${ }^{23,24}$

With regards to the distribution of patients by gender factor, more females than males were infected and the male to female infection ratio was 0.79:1, with no significant difference ( $\mathrm{p}>0.05$ ). Both males and females originating from rural areas were significantly more exposed to $\mathrm{CE}$ than urban residents $(\mathrm{p}<0.05$ ). The proportion of females to males with $\mathrm{CE}$ in any given group (age group, socio-geographic status and gender) is determined by factors increasing exposure to infection sources. These may include socio-economic and cultural status, local customs, behavioural activities and habits. ${ }^{8}$ The study findings may be explained by similar involvement of men and women, especially in rural areas, as both of them work because of difficult living conditions. In fact, in many rural areas of Morocco, in addition to their domestic practices, females are involved in farming works. They share the man's occupations in field tasks such as irrigation, harvesting, shepherding and feeding animals including dogs. This matter makes them exposed to

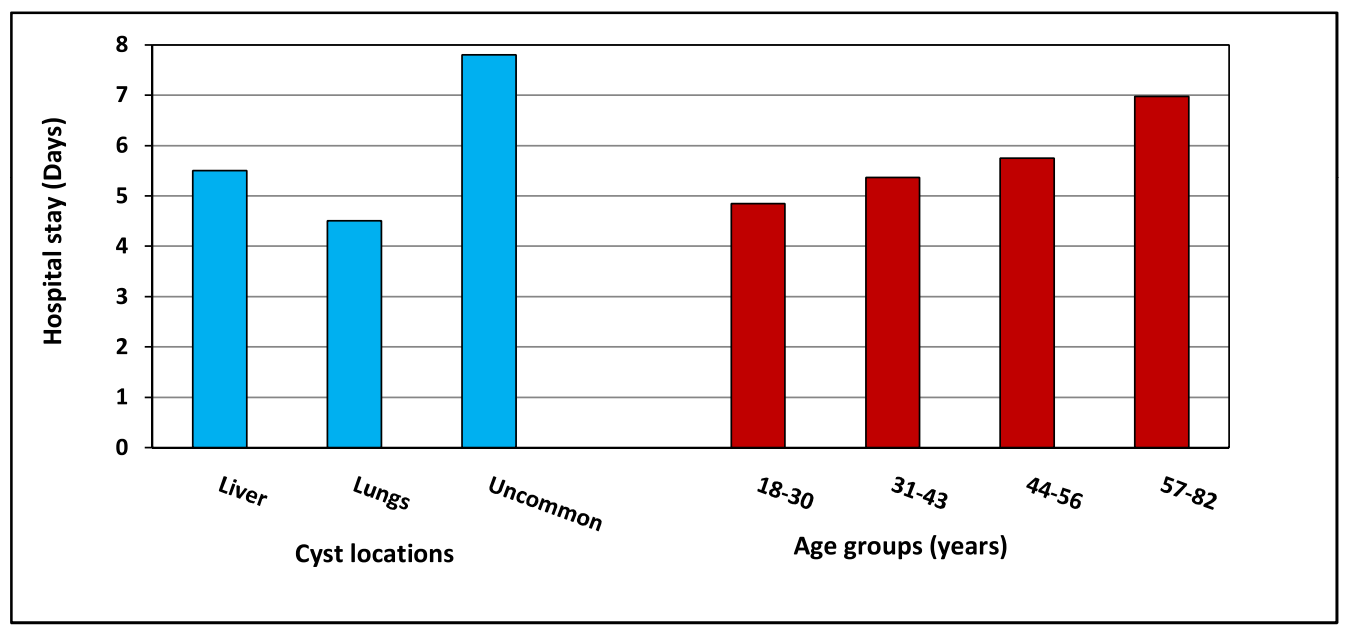

Fig. 3. Mean length of hospital stay according to age group and anatomic location of cysts in adults diagnosed and operated for cystic echinococcosis during the study period (2010-2016). 
infection due to environmental contamination with the parasite eggs. Cooking behaviours like tasting of contaminated raw vegetables or foodstuffs before cooking, habit of geophagy in females during pregnancy and hygienically poor practices may be responsible for higher rates of infection in women. Previous studies in adults reported a predominance of CE in females. ${ }^{25,26}$ Nonetheless, the European Centre for Disease Prevention and Control reported that no significant differences were found in $\mathrm{CE}$ rates by gender with a male-to-female ratio of $0.98: 1 .^{27}$

For the age factor, adults from 18 to 30 and 31-43 years were significantly more affected than older age groups; in line with the previously reported studies. ${ }^{28,29}$ The people in these age groups are the most active in livestock rearing and agricultural practices increasing the exposure risk factor to CE. Additionally, they may be more likely to seek medical attention with the disease symptoms development. The relatively higher incidence of CE in the active age group limits their ability to perform their routine activities and thus contributes to further economic losses. Over 44 years of age, a decrease of CE rates was observed. Although, significant number of younger adults reported to hospital for treatment as compared to older age groups over 44 years old ( $p<0.01$ ), yet the duration of infection and appearance of symptoms in symptomatic adults cannot be ascertained, as the infection usually occurs years before appearing of symptoms. However, the life style changes occurring in older adults and changing activities with age tend to decrease the chance of being infected with CE. Further, it can also be explained by the chronic nature of $\mathrm{CE}$, as the infection is usually acquired in childhood developing very slowly, remaining asymptomatic for years, and in symptomatic subjects, symptoms may manifest in adult age. $^{6}$ For all the defined age groups, the highest frequencies were observed in adults living in rural areas.

For the localization of cysts, only single organ involvement was identified in the investigated adults. The most commonly affected organs were liver and lung with a highly significant rate for liver cysts ( $\mathrm{p}<0.001$ ). The liver to lung infection ratio was 2.18:1. In contrast to adults, previous studies among children found significantly lower liver to lung infection ratios. ${ }^{12}$ Uncommon anatomic locations were significantly rare $(6.9 \%)$ and no multiple organ involvement was detected in the investigated adults. These findings are consistent with other studies reporting that unusual locations are predominant in adults than children, while combined liver and lung cyst are relatively more preponderant in children. ${ }^{7,8}$

All of the anatomic locations were significantly more prevalent in adults from rural zones $(\mathrm{p}<0.001)$. For the gender factor, liver cysts were significantly more identified in females than males while no statistically significant difference was observed for lung and unusual locations ( $p>0.05$ ). So the global difference between genders was mainly a consequence of higher counts of hepatic cysts in females than in males which is in line with previously reported findings. ${ }^{26}$ Within adults age groups, there was no significant difference between 18-30 years and 31-43 years age groups regarding liver and lung locations rates $(p>0.05)$. However, a significant decrease was observed in older age groups $(\mathrm{p}<0.01)$. The recorded differences may be related to several factors. It has been reported that the parasite burden and clinical course of the disease depend on host-parasite interactions, associated with the intensity and duration of infection, the affected tissue or organ and the immunity of the host. ${ }^{30}$ Epidemiological, clinical, and pathological features of CE are highly variable. They depend on several factors such as age, gender, infection severity, cyst size and the involved organ. ${ }^{18}$ Other factors including the Echinococccus genotype have been suggested to have a role in cyst localization, growth and complications. $^{31}$

The mean duration of hospitalization among the investigated CE patients was 5.5 days. Increased periods, but not significant, were registered in patients with unusual locations of hydatid cysts and age group 57-82 years, in accordance with previously reported findings. ${ }^{28}$ Vlad et al. ${ }^{12}$ found that the hospitalization period was similar in children (13.4 days, range of 2-49) and adult cases (14.1 days, range of 1-65). Male adults required significantly longer hospital stay (15.2 days, range of 2-65) as compared with female adults (13.1 days, range of 1-58). Longer mean hospital stay periods reaching more than 25 days have been reported by other authors. ${ }^{29}$ Further investigations indicated that the length of hospital may differ according to the complicated or uncomplicated status of the cysts and based on the treatment techniques and procedures. ${ }^{32}$

\section{Conclusion}

The undertaken study allowed the assessment of CE distribution in adults as well as factors leading to increased exposure risk and infection transmission. The occurrence of the infection indicates contamination of the environment with parasite eggs and active transmission of the parasitic infection from the main sources to humans, particularly in younger adults in rural areas. The pattern and features of the infection in adults' population should be considered in the prevention and control programs. In order to gain better insight into the problem with this zoonosis, additional epidemiological studies in children and adults, other intermediate hosts and definitive hosts need to be accomplished in $\mathrm{CE}$ endemic areas. In fact most epidemiologic reports based on hospital-based surveys, may underestimate the actual rates of infection, especially in low socio-economic groups with limited resources. In this regard, decentralizing diagnostic through surveys involving mass screening in rural areas, especially using ultrasound exams, can provide valuable information of the infection occurrence and allow a better understanding of risk factors that persist in rural populations. This may help to make more effective the inter-sectoral surveillance and control program implemented in the country, targeting humans, dogs and livestock populations, normalizing of slaughter facilities and epidemiological surveillance. Moreover, Health education is of paramount importance to increase populations' participation and to ensure maintenance of the long-term continuation for CE control in the studied region. This requires a long-term inter-sectoral collaboration between public health, agricultural, veterinary and local authorities as well as scientists to achieve significant decrease in CE occurrence or eradication.

\section{Financial support}

This research did not receive any specific grant from funding agencies in the public, commercial, or not-for-profit sectors.

\section{Declaration of competing interest}

The authors declare that there is no conflict of interest.

\section{Acknowledgements}

The authors are extremely grateful to the officials and staff of the Regional Direction of Health Marrakech-Safi and the Provincial Ambulatory Outreach Service (SIAAP -Marrakesh), for their valuable collaboration on this study. The assistance of Mr. Mohamed Essarafy is greatly appreciated.

\section{References}

1. Craig PS, Budke CM, Schantz PM, Li T, Qiu J, et al. Human echinococcosis: a neglected disease? Trop Med Health. 2007;35(4):283-292.

2. Eckert J, Deplazes P. Biological, epidemiological, and clinical aspects of echinococcosis, a zoonosis of increasing concern. Clin Microbiol Rev. 2004;17(1):107-135.

3. Torgerson PR. The emergence of echinococcosis in Central Asia. Parasitology. 2013;140(3):1667-1673.

4. World Health Organization WHO. Investing to Overcome the Global Impact of Neglected Tropical Diseases: Third Report on Neglected Tropical Diseases 2015; 2015:191 Geneva.

5. Tamarozzi F, Rossi P, Galati F, et al. The Italian registry of cystic echinococcosis (RIEC): the first prospective registry with a European future. Euro Surveill. 
2015;20(18):1-6.

6. Sundarka MK, Bansal R, Talwar V, Jindal R, Saini M, Gupta HL. Disseminated hydatid cyst disease presenting as acute respiratory distress. J Indian Acad Clin Med. 2000;1(3):257-259.

7. Talaiezadeh AH, Maraghi S. Hydatid disease in children: a different pattern than adults. Pak J Med Sci. 2006;22(3):329-332.

8. Djuricic SM, Grebeldinger S, Kafka DI, Djan I, Vukadin M, Vasiljevic ZV. Cystic echinococcosis in children - the seventeen-year experience of two large medical centers in Serbia. Parasitol Int. 2010;59(2):257-261.

9. Sarkari B, Arefkhah N, Ghorbani F, et al. Seroprevalence of cystic echinococcosis and related risk factors for infection among children in a rural community in Fars Province, Southern Iran. Clin Epidemiol Glob Health CEGH. 2019. https://doi.org/10. 1016/j.cegh.2019.03.009.

10. Creţu CM, Codreanu RR, Mastalier B, et al. Albendazole associated to surgery or minimally invasive procedures for hydatid disease - how much and how long. Chirurgia. 2012;107(1):15-21.

11. Derfoufi O, Ngoh Akwa E, Elmaataoui A, et al. [Epidemiological profile of echinococcosis in Morocco from 1980 to 2008]. Ann Biol Clin. 2012;70(4):457-461 [Article in French].

12. Vlad DC, Neghina AM, Dumitrascu V, Marincu I, Neghina R, Calma CL. Cystic echinococcosis in children and adults: a seven-year comparative study in western Romania. Foodb Pathog Dis. 2013;10(2):189-195.

13. Irabedra P, Ferreira C, Sayes J, et al. Control programme for cystic echinococcosis in Uruguay. Mem Inst Oswaldo Cruz. 2016;111(6):372-377.

14. Fallah N, Rahmati K, Fallah M. Prevalence of human hydatidosis based on hospital records in Hamadan west of Iran from 2006 to 2013. Iran J Parasitol. 2017;12(3):453-460 2017.

15. El Berbri I, Ducrotoy MJ, Petavy AF, Fassi Fihri O, Shaw AP, et al. Knowledge, attitudes and practices with regard to the presence, transmission, impact, and control of cystic echinococcosis in Sidi Kacem province, Morocco. Infect Dis Poverty. 2015;4(48):1-12

16. Dakkak A, El Berbri I, Petavy AF, et al. Echinococcus granulosus infection in dogs in Sidi Kacem Province (north-West Morocco). Acta Trop. 2017;165:26-32

17. Veit P, Bilger B, Schad V, Schäfer J, Frank W, Lucius R. Influence of environmental factors on the infectivity of Echinococcus multilocularis eggs. Parasitology. 1995; 110(1):79-86.

18. Higuita NIA, Brunetti E, McCloskeyc C. Cystic echinococcosis. J Clin MicrobioL
2016;54(3):518-523

19. Azlaf R, Dakkak A. Epidemiological study of the cystic echinococcosis in Morocco. Vet Parasitol. 2006;137:83-93.

20. Brunetti E, Garcia HH, Junghanss T. Cystic echinococcosis: chronic, complex, and still neglected. PLoS Neglected Trop Dis. 2011;5:e1146.

21. Chakarova B, Kichukova K, Rasheva G, et al. A short overview of the cystic echinococcosis among people in Bulgaria and Stara Zagora region during the period 2006-2014. Trakia J Sci. 2015;13(2):110-116.

22. Romani ELS, Rodrigues-Silva R, Maldonado A, Machado-Silva JR, Gomes DC. Notes on human cases of cystic echinococcosis in Peru. Mem Inst Oswaldo Cruz, Rio de Janeiro. 2006;101(3):335-337.

23. Colovic Calovski I, Barac A, Golubovic Z, et al. Case-series study of hepatic echinococcal cysts in Serbia: viability of scolices, seropositivity and epidemiological characteristics. J Helminthol. 2018;92(2):161-167.

24. Oudni-M'rad M, M'rad S, Babba H. Molecular and epidemiology data on cystic echinococcosis in Tunisia. In: Rodriguez-Morales AJ, ed. Current Topics in Echinococcosis. Croatia: Intech publisher; 2015:55-74.

25. Gonlugur U, Ozcelik S, Gonlugur TE, et al. The retrospective annual surgical incidence of cystic echinococcosis in Sivas, Turkey. Zoonoses and Public Health. 2009;56(5):209-214.

26. Al-Qaoud KM, Craig PS, Abdel-Hafez SK. Retrospective surgical incidence and case distribution of cystic echinococcosis in Jordan between 1994 and 2000. Acta Trop. 2003;87:207-214.

27. European Centre for Disease Prevention and Control. Annual Epidemiological Report: Food and Waterborne Diseases and Zoonoses. Stockholm: ECDC; 2014:96.

28. Lupu MA, Dragomir A, Paduraru AA, Ritiu SA, Lazar F, et al. Cystic echinococcosis in adult hospitalized patients: a 10-year retrospective study in Western Romania. Int $J$ Infect Dis. 2018;73(S):299-300

29. Nourjah N, Sahba G, Baniardalani M, Chavshin A. Study of 4850 operated hydatidosis cases in Iran. Southeast Asian J Trop Med Public Health. 2004;35(S1):218-222.

30. Mangano VD, Modiano D. Host genetics and parasitic infections. Clin Microbiol Infect. 2014;20:1265-1275.

31. Moro P, Schantz PM. Cystic echinococcosis in the americas. Parasitol Int. 2006;55(Suppl):S181-S186.

32. Mastrandrea S, Stegelb G, Piseddua T, Leddaa S, Masala G. A retrospective study on burden of human echinococcosis based on hospital discharge records from 2001 to 2009 in Sardinia, Italy. Acta Trop. 2012;123(3):184-189. 